\title{
ON ASYMPTOTIC VALUES OF FUNCTIONS IN A POLYDISK DOMAIN AND BAGEMIHL'S THEOREM
}

\begin{abstract}
Asymptotic sets of functions in a polydisk domain of arbitrary connectivity are studied. We construct an example of such function, having preassigned asymptotic set. This result generalizes well-known examples, obtained by M. Heins and W. Gross for entire functions. Moreover, it is found out that not all results on asymptotic sets of functions in $\mathbb{C}$ can be extended to functions in $\mathbb{C}^{n}$. In particular, this fact is connected with the failure of Bagemihl's theorem on ambiguous points for functions in $\mathbb{R}^{n}, n \geq 3$.
\end{abstract}

Key words: asymptotic value, analytic set, ambiguous point

2010 Mathematical Subject Classification: 32A40, $26 B 99$

Let $D_{1}, \ldots, D_{n}$ be domains in $\mathbb{C}, D=D_{1} \times \cdots \times D_{n}, z_{0}=\left(z_{1}^{0}, \ldots, z_{n}^{0}\right) \in$ $\in \partial D$ be an accessible boundary point i.e., there exists an open $\operatorname{arc} \Gamma \subset D$ with endpoint $z_{0}$. Let $f$ be a function defined in $D$.

Definition 1. 1, Section 1.6, p.8], 2] We say that $a \in \overline{\mathbb{C}}$ is an asymptotic value of $f$ at the point $z_{0}$ if there exists an arc $\gamma_{a} \subset D$ with endpoint $z_{0}$ such that

$$
\lim _{a \ni z \rightarrow z_{0}} f(z)=a
$$

The arc $\gamma_{a}$ is called an asymptotic curve corresponding to the asymptotic value $a$. The set of all asymptotic values (or, briefly, the asymptotic set) of $f$ at the point $z_{0}$ is denoted by $\operatorname{As}\left(f, z_{0}\right)$.

Asymptotic sets were actively studied for entire functions and meromorphic functions in $\mathbb{C}$.

(C) Petrozavodsk State University, 2015

\section{(c)) EY-NC}


It is well known that for a nonconstant entire function this set is analytic (in the sense of Suslin) [3. This means that $\operatorname{As}(f, \infty)$ can be represented in the form

$$
A s(f, \infty)=\bigcup_{\left(n_{1}, n_{2}, \ldots\right)}\left\{A_{n_{1}} \cap A_{n_{1} n_{2}} \cap A_{n_{1} n_{2} n_{3}} \cap \ldots\right\}
$$

where $n_{k}, k=1,2, \ldots$, are integer numbers, $A_{n_{1} \ldots n_{k}}$ are closed sets and the union extends over all sequences $\left(n_{1}, n_{2}, \ldots\right)$ (see [4, p. 105], [5, p. 136] for details). From the Iversen theorem [6] (see also [1, ch. 1], [7, section 5.1, p. 171]) it follows that for such functions this set contains the infinity. Many articles are devoted to constructing the examples of functions, having prescribed asymptotic sets. W. Gross [8] constructed an entire function whose set of asymptotic values at the infinity is equal to $\overline{\mathbb{C}}$. M. Heins [9] proved that every analytic set containing the infinity is an asymptotic set of some entire function.

In [10] and [1] functions analytic in planar domains of arbitrary connectivity were considered. For such functions theorems of W. Gross and M. Heins were generalized. Here the case when $z_{0}$ belongs to an isolated boundary fragment was considered.

Definition 2. [12] A domain $D \subset \mathbb{C}$ has an isolated boundary fragment if one of the following conditions holds:

(I) There exist a continuum $K \subset \partial D$ and an open set $U$ such that $K \subset U$ and $(\partial D \backslash K) \cap U=\emptyset$.

(II) There exist a Jordan arc $\Gamma \subset \partial D$ with distinct ends $\xi, \eta$ and an open disc $B$ such that $\xi, \eta \in \partial B, \Gamma \backslash\{\xi, \eta\} \subset B$ and $(\partial D \backslash \Gamma) \cap B=\emptyset$.

(III) There exist a point $a \in \partial D$ and an open disc $B(a)$ centered at $a$ such that $(B(a) \backslash\{a\}) \cap \partial D=\emptyset$, i.e., $a$ is an isolated point of the set $\partial D$.

The continuum $K$ from (I), the arc $\Gamma$ from (II), and the point a from (III) are called isolated boundary fragments of $D$.

Theorem A. 11] Let $D \subset \mathbb{C}$ be a domain with isolated boundary fragment $T$. Let point $\zeta_{0}$ belong to this fragment. If this fragment has type (I), then we assume, in addition, that $\zeta_{0}$ is an accessible and it is an impression of some prime end of $D$. Let $A$ be an analytic set, $\infty \in A$. Then there exists an analytic function $f$ such that $A s\left(f, \zeta_{0}\right)=A$.

Remark. The fact that $\zeta^{0}$ is an impression of some prime end of $D$ ( $D$ may not be simply connected) means that $\zeta^{0}$ is an impression of some prime end of the simply connected domain $G \supset D, \partial G=T$. 
In this note Theorem $\mathrm{A}$ is extended to functions analytic in a polydisk domain $D$. We describe simple construction of such function with a preassigned asymptotic set.

Theorem 1. Let $k$ be a fixed natural number, $1 \leq k \leq n$. Suppose $D=D_{1} \times \cdots \times D_{n}$, where $D_{i}, 1 \leq i \leq n, i \neq k$, are arbitrary domains in $\mathbb{C}, D_{k} \subset \mathbb{C}$ is a domain with an isolated boundary fragment $T$. Suppose $z_{0}=\left(z_{1}^{0}, \ldots, z_{n}^{0}\right) \in \partial D$, moreover $z_{i}^{0}, i \neq k$, is either points of the domains $D_{i}$ or accessible boundary points of $D_{i}, z_{k}^{0} \in T$. If $T$ is a fragment of type (I), then we assume in addition that $z_{k}^{0}$ is an accessible from $D_{k}$ and it is an impression of some prime end of $D_{k}$. Let $A$ be an analytic set, containing the infinity. Then there exists an analytic in $D$ function $f$ such that $A s\left(f, z_{0}\right)=A$.

Proof. Consider the domain $D_{k} \subset \mathbb{C}$ and the point $z_{k}^{0} \in T$. By Theorem A there exists an analytic in $D_{k}$ function $F(z)$, possessing the property $\operatorname{As}\left(F, z_{k}^{0}\right)=A$. Let $a \in A$ and $\Gamma_{k}^{a}$ be an asymptotic curve, corresponding to the value $a$. This means that

$$
\lim _{\Gamma_{k}^{a} \ni z_{k} \rightarrow z_{k}^{0}} F\left(z_{k}\right)=a .
$$

We construct the analytic in $D$ function

$$
f(z)=f\left(z_{1}, \ldots, z_{n}\right) \stackrel{\text { def }}{=} F\left(z_{k}\right) .
$$

Denote by $\Gamma_{i}^{a}, 1 \leq i \leq n, i \neq k$, a curve in $D_{i}$ with endpoint $z_{i}^{0}$. Then for any curve $\gamma_{a}$ with endpoint $z_{0}, \gamma_{a} \subset \Gamma_{1}^{a} \times \cdots \times \Gamma_{n}^{a}$, we have

$$
\lim _{\gamma_{a} \ni z \rightarrow z_{0}}=\lim _{\Gamma_{k}^{a} \ni z_{k} \rightarrow z_{k}^{0}} F\left(z_{k}\right)=a
$$

for all $a \in A$. Therefore, $\operatorname{As}\left(f, z_{0}\right)=A$.

Remark. If we put $n=1$, we obtain Theorem A.

In the case $n=1$ the following fact takes place (see [11]): if card $A>1$, then the set of all points $z_{0}$ such that a function $f$ defined in a simply connected domain $D, z_{0} \in \partial D$, possesses the property

$$
\operatorname{As}\left(f, z_{0}\right)=A
$$

is at most countable. This fact follows easily from Bagemihl's theorem on ambiguous points. 
If a function $f$ is defined in a domain $D \subset \mathbb{R}^{n}, \Gamma \subset D, z_{0} \in \partial D \cap \bar{\Gamma}$, then the cluster set $C\left(f, z_{0}, \Gamma\right)$ of $f$ at the point $z_{0}$ along $\Gamma$ [1, ch. 1] is the set of all numbers $w \in \overline{\mathbb{C}}$ such that there exists a sequence $z_{n} \in \Gamma$, $z_{n} \underset{n \rightarrow \infty}{\longrightarrow} z_{0}$ and $f\left(z_{n}\right) \underset{n \rightarrow \infty}{\longrightarrow} w$.

Definition 3. 13, 1, ch 4.7] A point $z_{0} \in D$ is an ambiguous point of $f$ if there exist two $\operatorname{arcs} \Gamma_{1}$ and $\Gamma_{2}$ in $\Delta$ with endpoint $z_{0}$ such that

$$
C\left(f, z_{0}, \Gamma_{1}\right) \cap C\left(f, z_{0}, \Gamma_{2}\right)=\emptyset .
$$

Bagemihl proved in [13] (see also [1, ch 4.7]) that an arbitrary function in the open unit disk can have at most countable set of ambiguous points.

Unlike the case $n=1$, for $n \geq 2$ there exist functions $f$ analytic in a simply connected polydisk domain $D$ such that

$$
\operatorname{As}\left(f, z_{0}\right)=A, \quad(\operatorname{card} A>1)
$$

at an uncountable set of points $z_{0} \in \partial D$.

Suppose all domains $D_{k}$ in Theorem 1 be simply connected. Let $f_{0}(z)$ be the function constructed in the proof of Theorem 1 . Then for every point $z_{0}=\left(z_{1}^{0}, \ldots, z_{n}^{0}\right)$ from the conditions of Theorem 1 we have

$$
\operatorname{As}\left(f_{0}, z_{0}\right)=A \text {. }
$$

The set of all such points $z_{0}$ is uncountable.

This difference of the case $n \geq 2$ from the case $n=1$ is connected with the fact that Bagemihl's theorem is not true in $\mathbb{R}^{n}$ for $n \geq 3$. There are functions in Euclidean ball $\mathbb{B}^{n}=\left\{x \in \mathbb{R}^{n}:\|x\| \leq 1\right\}, n \geq 3$, with an uncountable set of ambiguous points. Examples are given in [14 -16]. One more example can be obtained using Theorem 1 . Take $D_{k}=\Delta$ and $A$ such that card $A \geq 2$. Let $f$ be the function constructed in Theorem 1, $g$ be a homeomorphism of $\mathbb{B}^{2 n}$ onto the polydisk $\Delta^{n}=\Delta \times \cdots \times \Delta$. Then the set of ambiguous points of the composition $f \circ \varphi$ is uncountable.

In [17] P. J. Rippon introduced a new definition of ambiguous point of function in $\mathbb{R}^{n}, n \geq 3$. He replaced one arc by the boundary of a subdomain of $\mathbb{B}^{n}$. More precisely, P. J. Rippon says that a point $z_{0} \in \partial \mathbb{B}^{n}$ is an ambiguous point of a function $f$ defined in $\mathbb{B}^{n}$ if there exist

1) a subdomain $S$ of $\mathbb{B}^{n}, \partial S \cap \partial \mathbb{B}^{n}=\left\{z_{0}\right\}$,

$2)$ an $\operatorname{arc} \Gamma \subset S$ with endpoint $z_{0}$

such that

$$
C\left(f, z_{0}, \Gamma\right) \cap C\left(f, z_{0}, \partial S \backslash\left\{z_{0}\right\}\right)=\emptyset .
$$


This definition allows to obtain the analog of Bagemihl's theorem in $\mathbb{R}^{n}$, $n \geq 3$ [17]: for any function in $\mathbb{B}^{n}$ the set of all ambiguous points (in the sense of Rippon) is at most countable.

One can pose the following problem: how to define the ambiguous point of function in $\mathbb{B}^{n}, n \geq 3$, using object of the same nature (like two arcs in Bagemihl's definition), saving the statement about countability of the set of such points. In view of Rippon's definition it is natural to consider points $z_{0} \in \partial \mathbb{B}^{n}$ for which there exist subdomains $S_{1}$ and $S_{2}$ of $\mathbb{B}^{n}, \partial S_{1} \cap \partial \mathbb{B}^{n}=\partial S_{2} \cap \partial \mathbb{B}^{n}=\left\{z_{0}\right\}$, and

$$
C\left(f, z_{0}, \partial S_{1} \backslash\left\{z_{0}\right\}\right) \cap C\left(f, z_{0}, \partial S_{2} \backslash\left\{z_{0}\right\}\right)=\emptyset .
$$

The following example shows that this assumption does not save Bagemihl's theorem true even for continuous functions.

Example. Take the function

$$
g(t)=\frac{|1-t|}{1-|t|}, t \in \Delta=\{z \in \mathbb{C}:|z|<1\}
$$

Construct our example, using the function $g(t)$. For $x=\left(x_{1}, \ldots, x_{n}\right) \in \mathbb{B}^{n}$ put

$$
\begin{aligned}
f(x) & =\frac{\sqrt{\left(1-\frac{x_{1}}{\sqrt{1-x_{3}^{2}-\cdots-x_{n}^{2}}}\right)^{2}+\frac{x_{2}^{2}}{1-x_{3}^{2}-\cdots-x_{n}^{2}}}}{1-\sqrt{\frac{x_{1}^{2}+x_{2}^{2}}{1-x_{3}^{2}-\cdots-x_{n}^{2}}}}= \\
= & \frac{\left|1-\left(\frac{x_{1}}{\sqrt{1-x_{3}^{2}-\cdots-x_{n}^{2}}}+i \frac{x_{2}}{\sqrt{1-x_{3}^{2}-\cdots-x_{n}^{2}}}\right)\right|}{1-\left|\frac{x_{1}}{\sqrt{1-x_{3}^{2}-\cdots-x_{n}^{2}}}+i \frac{x_{2}}{\sqrt{1-x_{3}^{2}-\cdots-x_{n}^{2}}}\right|}=g(t),
\end{aligned}
$$

where

$$
t=\frac{x_{1}}{\sqrt{1-x_{3}^{2}-\cdots-x_{n}^{2}}}+i \frac{x_{2}}{\sqrt{1-x_{3}^{2}-\cdots-x_{n}^{2}}} \in \Delta .
$$

Consider the set $l=\partial \mathbb{B}^{n} \cap\left\{\left(x_{1}, 0, x_{3}, \ldots, x_{n}\right), x_{1}>0, x_{3}, \ldots, x_{n} \in \mathbb{R}\right\}$. Let

$$
\zeta=\left(\zeta_{1}, 0, \zeta_{3}, \ldots, \zeta_{n}\right) \in l
$$


Fix $\varepsilon \in(0 ; \pi / 4)$. By $\Pi$ denote the plane

$$
\left\{\begin{array}{l}
x_{3}=\zeta_{3} \\
\cdots \\
x_{n}=\zeta_{n}
\end{array}\right.
$$

Let $\gamma_{0}$ be the segment $\left[\left(0,0, \zeta_{3}, \ldots, \zeta_{n}\right),\left(\zeta_{1}, 0, \zeta_{3}, \ldots, \zeta_{n}\right)\right] \subset \Pi$. By $\gamma_{\frac{\pi}{4}-\varepsilon}$ denote the segment $\left[\left(\zeta_{1}, 0, \zeta_{3}, \ldots, \zeta_{n}\right),\left(0, \zeta_{1} \operatorname{tg}\left(\frac{\pi}{4}-\varepsilon\right), \zeta_{3}, \ldots, \zeta_{n}\right)\right]$. Consider the open triangle $T_{\frac{\pi}{4}-\varepsilon} \subset \Pi$ bounded by the segments $\gamma_{0}, \gamma_{\frac{\pi}{4}-\varepsilon}$ and the ray $\left\{\left(0, x_{2}, \zeta_{3}, \ldots, \zeta_{n}^{4}\right), x_{2}>0\right\}$.

Let $S_{1}$ be a subdomain of $\mathbb{B}^{n}$ such that

1.1) $\partial S_{1} \cap \partial \mathbb{B}^{n}=\{\zeta\}$,

1.2) for each $x=\left(x_{1}, \ldots, x_{n}\right) \in S_{1}$ the projection $\widetilde{x}=\left(x_{1}, x_{2}, \zeta_{3}, \ldots, \zeta_{n}\right)$ of $x$ to $\Pi$ belongs to $T_{\frac{\pi}{4}-\varepsilon}$,

1.3) $\left|x_{k}\right|<\left|\zeta_{k}\right|, k=3, \ldots, n$ for each $x=\left(x_{1}, \ldots, x_{n}\right) \in S_{1}$.

Suppose $p \in C\left(f, \zeta, \partial S_{1} \backslash\{\zeta\}\right)$. This means that there exists a sequence $w^{N}=\left(w_{1}^{N}, \ldots, w_{n}^{N}\right) \in \partial S_{1}, w^{N} \rightarrow \zeta$ as $N \rightarrow \infty$, such that $f\left(w^{N}\right) \rightarrow p$ as $N \rightarrow \infty$. Using (1), for each point $w^{N} \in \mathbb{R}^{n}$ let us construct the point $t^{N} \in \Delta$, substituting $x_{k}$ by $w_{k}^{N}$ in $(1)$. Then we get $t^{N} \rightarrow 1$ as $N \rightarrow \infty$. By $\beta\left(t^{N}\right)$ denote the angle between the segments $\left[t^{N} ; 1\right]$ and $[0 ; 1]$ in $\Delta$. By condition 1.3) we have

$$
\sqrt{1-\left(w_{3}^{N}\right)^{2}-\cdots-\left(w_{n}^{N}\right)^{2}} \geq \sqrt{1-\zeta_{3}^{2}-\cdots-\zeta_{n}^{2}}
$$

Therefore,

$$
\begin{aligned}
& 0 \leq \operatorname{Re} t^{N}=\frac{w_{1}^{N}}{\sqrt{1-\left(w_{3}^{N}\right)^{2}-\cdots-\left(w_{n}^{N}\right)^{2}}} \leq \frac{w_{1}^{N}}{\sqrt{1-\zeta_{3}^{2}-\cdots-\zeta_{n}^{2}}}, \\
& 0 \leq \operatorname{Im} t^{N}=\frac{w_{2}^{N}}{\sqrt{1-\left(w_{3}^{N}\right)^{2}-\cdots-\left(w_{n}^{N}\right)^{2}}} \leq \frac{w_{2}^{N}}{\sqrt{1-\zeta_{3}^{2}-\cdots-\zeta_{n}^{2}}} .
\end{aligned}
$$

From condition 1.2) it follows that the point

$$
\frac{w_{1}^{N}+i w_{2}^{N}}{\sqrt{1-\zeta_{3}^{2}-\cdots-\zeta_{n}^{2}}}=\frac{w_{1}^{N}+i w_{2}^{N}}{\zeta_{1}}
$$

belongs to the triangle $\Delta_{\frac{\pi}{4}-\varepsilon} \stackrel{\text { def }}{=} \frac{1}{\zeta_{1}} \cdot T_{\frac{\pi}{4}-\varepsilon} \subset \Delta$. Consequently, taking into account (2) and (3), we obtain that $t^{N}$ belongs to $\Delta_{\frac{\pi}{4}-\varepsilon}$ too. Hence,

$$
0 \leq \beta\left(t^{N}\right) \leq \frac{\pi}{4}-\varepsilon
$$


for all $w^{N} \in \partial S_{1}$.

Denoting $a=\left|1-t^{N}\right|$, we have

$$
\frac{\left|1-t^{N}\right|}{1-\left|t^{N}\right|}=\frac{a}{1-\sqrt{a^{2}+1-2 a \cos \beta\left(t^{N}\right)}} \leq \frac{a}{1-\sqrt{a^{2}+1-2 a \cos \left(\frac{\pi}{4}-\varepsilon\right)}} .
$$

Then

$$
\begin{aligned}
& p=\lim _{N \rightarrow \infty} f\left(w^{N}\right)=\lim _{N \rightarrow \infty} g\left(t^{N}\right)=\lim _{N \rightarrow \infty} \frac{\left|1-t^{N}\right|}{1-\left|t^{N}\right|} \leq \\
& \leq \lim _{a \rightarrow 0} \frac{a}{1-\sqrt{a^{2}+1-2 a \cos \left(\frac{\pi}{4}-\varepsilon\right)}}=\frac{1}{\cos \left(\frac{\pi}{4}-\varepsilon\right)} .
\end{aligned}
$$

Now consider a domain $S_{2} \subset \mathbb{B}^{n}$, possessing the following properties:

2.1) $\partial S_{2} \cap \partial \mathbb{B}^{n}=\{\zeta\}$,

2.2) for every $x=\left(x_{1}, \ldots, x_{n}\right) \in S_{2}$ the projection $\widetilde{x}=\left(x_{1}, x_{2}, \zeta_{3}, \ldots, \zeta_{n}\right)$ of $x$ to $\Pi$ belongs to the set $T_{\frac{\pi}{4}+\varepsilon}$, bounded by the circle $\partial \mathbb{B}^{n} \cap \Pi$ and the straight line, passing through the points $\left(\zeta_{1}, 0, \zeta_{3}, \ldots, \zeta_{n}\right)$ and $\left(0, \zeta_{1} \operatorname{tg}\left(\frac{\pi}{4}+\right.\right.$ $\left.+\varepsilon), \zeta_{3}, \ldots, \zeta_{n}\right) ;\left(0,0, \zeta_{3}, \ldots, \zeta_{n}\right) \notin T_{\frac{\pi}{4}+\varepsilon}$.

2.3) $\left|x_{k}\right|>\left|\zeta_{k}\right|, k=3, \ldots, n$, for each $x=\left(x_{1}, \ldots, x_{n}\right) \in S_{2}$.

If $p^{\prime} \in C\left(f, \zeta, \partial S_{2} \backslash\{\zeta\}\right)$, then there exists a sequence $\omega^{N}=\left(\omega_{1}^{N}, \ldots, \omega_{n}^{N}\right) \in$ $\in \partial S_{2}, \omega^{N} \rightarrow \zeta$ as $N \rightarrow \infty$, such that $f\left(\omega^{N}\right) \rightarrow p^{\prime}$. For $\omega^{N} \in \mathbb{R}^{n}$ calculate $\tau^{N} \in \Delta$ by formula (1). By 2.3),

$$
\sqrt{1-\left(\omega_{3}^{N}\right)^{2}-\cdots-\left(\omega_{n}^{N}\right)^{2}} \leq \sqrt{1-\zeta_{3}^{2}-\cdots-\zeta_{n}^{2}}
$$

Consequently,

$$
\begin{aligned}
\operatorname{Re} \tau^{N} & =\frac{\omega_{1}^{N}}{\sqrt{1-\left(\omega_{3}^{N}\right)^{2}-\cdots-\left(\omega_{n}^{N}\right)^{2}}} \geq \frac{\omega_{1}^{N}}{\sqrt{1-\zeta_{3}^{2}-\cdots-\zeta_{n}^{2}}}, \\
\operatorname{Im} \tau^{N} & =\frac{\omega_{2}^{N}}{\sqrt{1-\left(\omega_{3}^{N}\right)^{2}-\cdots-\left(\omega_{n}^{N}\right)^{2}}} \geq \frac{\omega_{2}^{N}}{\sqrt{1-\zeta_{3}^{2}-\cdots-\zeta_{n}^{2}}} .
\end{aligned}
$$

Since, by condition 2.2),

$$
\frac{\omega_{1}^{N}+i \omega_{2}^{N}}{\sqrt{1-\zeta_{3}^{2}-\cdots-\zeta_{n}^{2}}} \in \Delta_{\frac{\pi}{4}+\varepsilon} \stackrel{\text { def }}{=} \frac{1}{\zeta_{1}} T_{\frac{\pi}{4}+\varepsilon}
$$


we have $\tau^{N} \in \Delta_{\frac{\pi}{4}+\varepsilon}$. This yields that $\beta\left(\tau^{N}\right) \geq \frac{\pi}{4}+\varepsilon$ for all $N \in \mathbb{N}$. Hence, denoting $b=\left|1-\tau^{N}\right|$, we get

$$
\frac{\left|1-\tau^{N}\right|}{1-\left|\tau^{N}\right|}=\frac{b}{1-\sqrt{b^{2}+1-2 b \cos \beta\left(t^{N}\right)}} \geq \frac{b}{1-\sqrt{b^{2}+1-2 b \cos \left(\frac{\pi}{4}+\varepsilon\right)}} .
$$

This implies that

$$
p^{\prime}=\lim _{N \rightarrow \infty} f\left(\omega^{N}\right) \geq \lim _{b \rightarrow 0} \frac{b}{1-\sqrt{b^{2}+1-2 b \cos \left(\frac{\pi}{4}+\varepsilon\right)}}=\frac{1}{\cos \left(\frac{\pi}{4}+\varepsilon\right)} .
$$

We have proved that for all $p \in C\left(f, \zeta, \partial S_{1} \backslash\{\zeta\}\right)$ and $p^{\prime} \in C\left(f, \zeta, \partial S_{2} \backslash\right.$ $\backslash\{\zeta\})$ the following inequality holds

$$
p \leq \frac{1}{\cos \left(\frac{\pi}{4}-\varepsilon\right)}<\frac{1}{\cos \left(\frac{\pi}{4}+\varepsilon\right)} \leq p^{\prime} .
$$

This yields

$$
C\left(f, \zeta, \partial S_{1} \backslash\{\zeta\}\right) \cap C\left(f, \zeta, \partial S_{2} \backslash\{\zeta\}\right)=\emptyset
$$

for each $\zeta \in l$. The set of all points, possessing property (5), contains $l$. Therefore this set is uncountable.

Remark. The above example shows that the requirement for $\Gamma$ to be a subset of $D$ in Rippon's definition is essential. If we take $D=S_{1}$ from the example and $\Gamma$ be a curve, contained in the domain $S_{2}$, then the function $f$ has the property

$$
C(f, \zeta, \Gamma) \cap C(f, \zeta, \partial D \backslash\{\zeta\})=\emptyset
$$

at uncountable set of points $\zeta$.

Acknowledgment. This work was partially supported by a grant from the Simons Foundation and RFBR (project N 14-01-00510a).

\section{References}

[1] Collingwood E. F., Lohwater A. J. The theory of cluster sets. Cambridge Univ. Press, 1966.

[2] Hazewinkel M. Encyclopaedia of Mathematics. Vol. 1: AB, Kluwer Academic, Dordrecht, 1988. 
[3] Mazurkiewicz S. Sur les points singuliers d'une fonction analytique. Fund. Math., 1931, vol. 17, is. 1, pp. 26-29.

[4] Hausdorff F. Set theory. AMS Chelsea Publishing, 1957.

[5] Sierpinski M. General topology. University of Toronto Press, 1952.

[6] Iversen F. Recherches sur les fonctions inverses des fonctions méromorphes. Imprimerie de la Société de littérature finnoise, Helsinki, 1914.

[7] Goldberg A. A., Ostrovskii I. V. Value distributions of meromorphic functions. American Mathematical Society, Providence, R. I., 2008.

[8] Gross W. Eine ganze Funktion für die jede Komplexe Zahl Konvergenzwert ist. Math. Ann., 1918, vol. 79, pp. 201-208.

[9] Heins M. The set of asymptotic values of an entire function. Proceedings of the Scandinavian Math. Congress (Lund 1953), 1954, pp. 56-60.

[10] Ganenkova E. G., Starkov V. V. Asymptotic values of functions, analytic in planar domains. Probl. Anal. Issues Anal., 2013, vol. 2(20), no. 1, pp. 3842. DOI: $10.15393 / \mathrm{j} 3$.art.2013.2341.

[11] Ganenkova E. G., Starkov V. V. Analytic in planar domains functions with preassigned asymptotic set. J. Appl. Anal., 2014, vol. 20, is. 1, pp. 7-14. DOI: $10.1515 /$ jaa-2014-0002.

[12] Liczberski P., Starkov V. V. On locally biholomorhic mappings from multiconnected onto simply connected domains. Ann. Polon. Math., 2005, vol. 85, no. 2, pp. 135-143. DOI: 10.1016/j.jmaa.2008.11.067.

[13] Bagemihl F. Curvilinear cluster sets of arbitrary functions. Proc. Natl. Acad. Sci. USA, 1955, vol. 41, no. 6, pp. 379-382.

[14] Piranian G. Ambiguous points of a function continuous inside a sphere. Michigan Math. J., 1957, vol. 4, is. 2, pp. 151-152.

[15] Bagemihl F. Ambiguous points of a function harmonic inside a sphere. Michigan Math. J., 1957, vol. 4, is. 2, pp. 153-154.

[16] Church P.T. Ambiguous points of a function homeomorphic inside a sphere. Michigan Math. J., 1957, vol. 4, is. 2, pp. 155-156.

[17] Rippon P.J. Ambiguous points of functions in the unit ball of euclidean space. Bull. Lond. Math. Soc., 1983, vol. 15, no. 4, pp. 336-338. DOI: 10.1112/blms/15.4.336.

Received December 10, 2015.

Petrozavodsk State University

33, Lenina st., 185910 Petrozavodsk, Russia

E-mail: g_ek@inbox.ru 